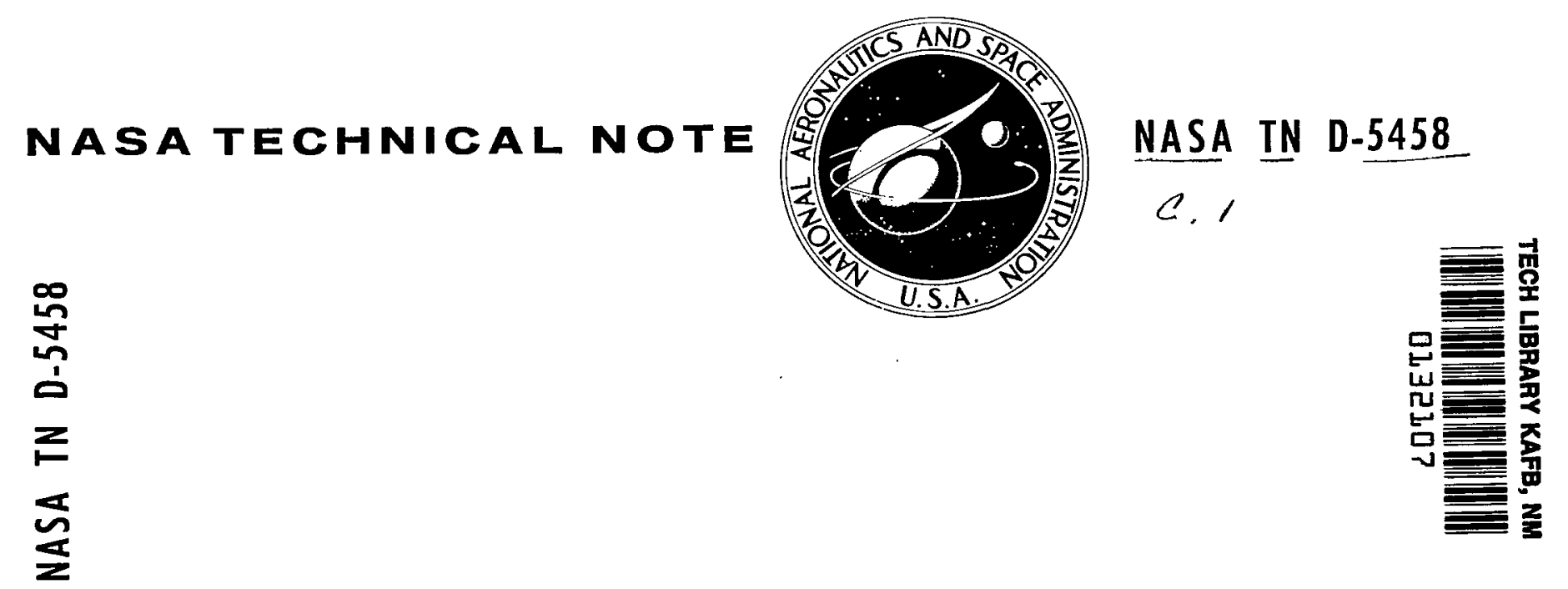

IOAN COPY: RETURN TO

AFWL (WLOL)

KIRTIMND AFB, N UERX

\title{
THE SCATTERING OF POLARIZED LIGHT BY POLYDISPERSE SYSTEMS OF IRREGULAR PARTICLES
}

by Alfred C. Holland

Electronics Research Center

Cambridge, Mass.

NATIONAL AERONAUTICS AND SPACE ADMINISTRATION - WASHINGTON, D. C. - NOVEMBER 1969 
1. Report No. NASA TIN D-5458

4. Title and Subtitle

The Scattering of Polarized Iight by Polydisperse Systems of Irregular Particles

7. Author(s)

Aifred C. Holland

9. Performing Organizotion Name and Adóress

Electronics Research Center Cambridge, Mass.

12. Sponsoring Agency Nome and Address

National Aeronautics and Space

Administration

Washington, D.C. 20546

15. Supplementary Notes

16. Abstract

The elements of the Mueller matrix for polydisperse systems of irregular, randomly oriented particles have been measured in absolute terms as a function of scattering angle for one wavelength. These results have been compared to the matrix elements that were calculated for assemblies of spherical particles that fit the same particle size distribution function and have the same (real) refractive index. Correlations between the measured and calculated matrix elements are discussed.

17. Key Words. Mueller Matrix

- Polydisperse Systems

- Spherical Particles

- Refractive Index
18. Distribution Statement

Unclassified - Unlimited

19. Security Classif. (of this ropart) Unclassified

20. Security Classif, (of this page) Unclassified

21. No, of Pages

23
22. Price $*$ $\$ 3.00$

*For sale by the Clearinghouse for Federal Scientific and Technical Information Springfield, Virginia 22151 


\section{THE SCATTERING OF POLARIZED LIGHT BY POLYDISPERSE SYSTEMS OF IRREGULAR PARTICLES}

By Alfred C. Holland

Electronics Research Center

\section{SUMMARY}

The elements of the Mueller matrix for polydisperse systems of irregular, randomly oriented particles have been measured in absolute terms as a function of scattering angle for one wavelength. These results have been compared to the matrix elements that were calculated for assemblies of spherical particles that fit the same particle size distribution function and have the same (real) refractive index. Correlations between the measured and calculated matrix elements are discussed.

\section{I.- INTRODUCTION}

The atmospheric aerosols exert a profound influence on the optical properties of the earth's atmosphere. Despite their low concentration relative to the molecular constituents, the aerosol's effect on atmospheric scattering can never be completely ignored, particularly when polarization phenomena are considered. The atmospheric aerosol is composed of particles of many different shapes, sizes, and materials. Most of the aerosol particles (dusts, smokes, snow, and ice crystals) are known to have nonspherical shapes.

Before a complete description of light scattering by the atmosphere can be made, one must know the detailed scattering behavior of such nonspherical particles: specifically, how the scattering matrix of the atmospheric aerosol depends on the particle composition, size distribution, shape, and, finally, on the wavelength of the incident light. However, except for spherical particles, and within certain limits, ellipsoids and cylinders, no rigorous theoretical description of particulate scattering is known.

The practical importance of the problem can hardly be overestimated. Beyond the case of atmospheric optics and the effect of the atmosphere on solar insolation and, hence, climatology, there is the possibility of using light beams to probe the atmosphere. Determining the properties of an unknown medium by examining the behavior of its scattering matrix with scattering angle and wavelength has many potential applications.

At the present time, most investigators (for lack of any better practical model) assume that their scattering particles 
are spheres. The scattering properties of spheres can be calculated exactly with a large digital computer.

Deirmendjian (ref. 1) for example, has calculated scattering matrix elements for models of haze and fog and found his results to be in excellent agreement with the measurements of pritchard and Elliott on natural fog (ref. 2).

There has been some experimental evidence that the scattering behavior of assemblies of irregular but randomly-oriented particles is similar to the scattering behavior of assemblies of spherical particles. Hodkinson (ref. 3) showed that the angular distribution of light scattered in the forward direction by samples of quartz, flint, and diamond dusts was similar to the light distribution predicted on the basis of geometric optics. Holland and Draper (ref. 4) showed reasonably good agreement between the unpolarized scattering coefficient for polydisperse samples of talc and that calculated for spherical particles fitting the same size distribution.

The experiments reported here have two main objectives. The first objective is to investigate possible correlations between the scattering matrix elements of irregular (but randomly oriented) particles and the matrix elements calculated for spherical particles having the same size distribution and refractive index. The second objective is to compile sufficient data on the scattering properties of irregular particles of known size distribution and physical properties to aid in the development or testing of the necessary theoretical models.

Considering the second objective, this work can be viewed as a preliminary step in a program aimed at developing methods for describing the scattering of light by real media.

\section{II. - THEORETICAI CONSIDERATIONS}

The scattering of a polarized beam of light by an optically thin volume element of a scattering medium can be described efficiently by the Mueller calculus. The scattering event is described as a Iinear transformation; the stokes vector of the incident light beam is transformed into the stokes vector of the scattered beam by the Mueller or scattering matrix:

$$
\left\{\Gamma_{i}\right\}=\text { const }\left[s_{i j}\right]\left\{\Gamma_{j}\right\} \text {. }
$$

The stokes vectors of both incident and scattered beams depend only on the characteristics of the two beams, while the scattering matrix depends only on the characteristics of the scattering medium. 
The stokes parameters and the Mueller matrix have been discussed fully by many authors including Perrin (ref. 5), Van De Hulst (ref. 6), Rozenberg (ref. 7), and Parke (ref. 8) and will not be described in detail here. In brief, if one chooses as the reference plane the scattering plane, defined by the directions of the incident and the scattered beams, then the complex electric vector of either beam can be decomposed into components perpendicular and parallel to the reference plane. With this convention, the stokes parameters are defined as:

$$
\begin{aligned}
& I=\left\langle E_{\ell} E_{\ell}^{*}+E_{r} E_{r}^{*}\right\rangle \\
& Q=\left\langle E_{\ell} E_{\ell}^{*}-E_{r} E_{r}^{*}\right\rangle \\
& U=\left\langle 2 R_{e}\left\{E_{\ell} E_{r}^{*}\right\}\right\rangle=\left\langle E_{\ell} E_{r}^{*}+E_{r} E_{\ell}^{*}\right\rangle \\
& V=\left\langle-2 \operatorname{Im}\left\{E_{\ell} E_{r}^{*}\right\}\right\rangle=\left\langle i\left(E_{\ell} E_{r}^{*}+E_{r} E_{\ell}^{*}\right)\right\rangle
\end{aligned}
$$

The subscripts $l$ and $r$ denote components parallel and perpendicular to the reference plane; the brackets denote time averages of the noted quantities, and the asterisks denote complex conjugates.

The stokes parameters all have the dimensions of intensity and satisfy the following inequality:

$$
\mathrm{I}^{2} \geq \mathrm{Q}^{2}+\mathrm{U}^{2}+\mathrm{V}^{2}
$$

The equality holds only for completely polarized light. It can be shown (ref. 9) that this characterization of a light beam is unique and that two beams of light with identical stokes parameters cannot be distinquished by optical analysis.

The four stokes parameters can be considered as components of a four-vector and a scattering event described as a matrix multiplication:

$$
\left[\begin{array}{l}
I_{s} \\
Q_{s} \\
U_{s} \\
v_{s}
\end{array}\right]=\frac{I}{k^{2}}\left[\begin{array}{lll} 
& s_{i j} \\
&
\end{array}\right]\left[\begin{array}{l}
I_{0} \\
Q_{0} \\
U_{0} \\
v_{0}
\end{array}\right]
$$

In general, the scattering matrix has sixteen independent coefficients; however, Perrin has shown that if the scattering 
medium is isotropic and free of optical activity the scattering matrix has only six independent non-zero coefficients:

$$
\left[s_{i j}\right]=\left[\begin{array}{cccc}
s_{11} & s_{12} & 0 & 0 \\
s_{12} & s_{22} & 0 & 0 \\
0 & 0 & s_{33} & s_{34} \\
0 & 0 & -s_{34} & s_{44}
\end{array}\right]
$$

The form of the matrix for different types of media has been discussed in detail by Van De Hulst (ref. 6). Rozenberg (ref. 7) points out that in general the matrix elements depend not only on the material properties of the medium but on the scattering angle $\theta$ measured in the scattering plane and on $\phi$ the azimuth of the scattering plane itself from some reference direction. Only in the case of an isotropic medium can one define matrix elements that are independent of the azimuth angle $\phi$ and that depend only on the usual scattering angle.

For a single homogeneous isotropic sphere we have the additional simplification $S_{11}=S_{22}$ and $S_{33}=S_{44}$. There are, then, only four independent non-zero matrix elements. These four coefficients can be expressed in terms of the intensity functions $i_{j}(\tilde{m}, x, \theta),(j=1,2,3,4)$ and the scattering efficiency $Q_{s}(\tilde{m}, x)$ obtained from the Mie theory:

$$
\begin{aligned}
& s_{11}(\tilde{m}, x, \theta)=s_{22}(\tilde{m}, x, \theta)=\frac{1}{2}\left\{_{i}(\tilde{m}, x, \theta)+i_{1}(\tilde{m}, x, \theta)\right\} \\
& S_{12}(\tilde{\mathrm{m}}, \mathrm{x}, \theta)=\mathrm{S}_{21}(\tilde{\mathrm{m}}, \mathrm{x}, \theta)=\frac{1}{2}\left\{\mathrm{i}_{2}(\tilde{\mathrm{m}}, \mathrm{x}, \theta)-\mathrm{i}_{1}(\tilde{\mathrm{m}}, \mathrm{x}, \theta)\right\} \\
& S_{33}(\tilde{m}, x, \theta)=S_{44}(\tilde{m}, x, \theta)=i_{3}(\tilde{m}, x, \theta) \\
& S_{43}=-S_{34}=i_{4}(\tilde{m}, x, \theta)
\end{aligned}
$$

The matrix elements defined above hold for a single spherical particle of refractive index $\tilde{m}$ and Mie size parameter $\mathrm{x}=\pi \mathrm{D} / \lambda$. The parameter $D$ is the diameter of the particle and $\lambda$ is the wavelength of the incident light.

For a polydisperse collection of spherical particles whose diameters are distributed according to a frequericy function $f(D) d D$ (particles/ $\mathrm{cm}^{3}-\mathrm{cm}$ ), one can define comparable volume intensity functions as: 


$$
\frac{P_{j}\left(\tilde{m}, x_{o}, \theta\right)}{4 \pi}=\frac{1}{k^{2} \beta_{s}(\tilde{m}, x)} \int_{x_{a}}^{x_{M}} f(x) i_{j}(\tilde{m}, x, \theta) d x \quad \quad(j=1,2,3,4)
$$

The volume scattering coefficient is defined in terms of the Mie scattering efficiency:

$$
\beta_{S}(\tilde{m}, x)=\frac{\lambda^{2}}{4 \pi} \int_{x_{a}}^{x_{M}} x^{2} Q_{S}(m, x) f(x) d x .
$$

In the expression above we have used $\mathrm{x}_{a}=\pi \mathrm{a} / \lambda, \mathrm{x}_{\mathrm{M}}=\pi \mathrm{D}_{\mathrm{M}} / \lambda$; $k=2 \pi / \lambda$ is the optical wave number or propagation constant. The quantity $x_{0}$ is the Mie size parameter corresponding to the median particle diameter of the size distribution; $\mathrm{x}_{\mathrm{O}}=\pi \mathrm{D}_{\mathrm{O}} / \lambda$. With these definitions, the scattering matrix for a volume element of homogeneous, isotropic spheres that fit the distribution function is:

$$
\left[s_{i j}\right]=B_{s}\left(\tilde{m}, x_{0}\right)\left[\begin{array}{cccc}
\frac{1}{2}\left[P_{2}\left(\tilde{m}, x_{0}, \theta\right)+P_{1}\left(\tilde{m}, x_{0}, \theta\right)\right] \frac{1}{2}\left[P_{2}\left(\tilde{m}, x_{0}, \theta\right)-P_{1}\left(\tilde{m}, x_{0}, \theta\right)\right] & 0 & 0 \\
\frac{1}{2}\left[P_{2}\left(\tilde{m}, x_{0}, \theta\right)-P_{1}\left(\tilde{m}, x_{0}, \theta\right)\right] \frac{1}{2}\left[P_{2}\left(\tilde{m}, x_{0}, \theta\right)+P_{1}\left(\tilde{m}, x_{0}, \theta\right)\right] & 0 & 0 \\
0 & 0 & \frac{P_{3}\left(\tilde{m}, x_{0}, \theta\right)}{4 \pi} & \frac{P_{4}\left(\tilde{m}, x_{0}, \theta\right)}{4 \pi} \\
0 & 0 & \frac{-P_{4}\left(\tilde{m}, x_{0}, \theta\right)}{4 \pi} & \frac{P_{3}\left(\tilde{m}, x_{0}, \theta\right)}{4 \pi}
\end{array}\right]
$$

The matrix elements are normalized so that

$$
\int \frac{1}{2}\left\{\frac{\mathrm{P}_{2}\left(\tilde{\mathrm{m}}, \mathrm{x}_{\mathrm{O}^{\prime}}, \theta\right)}{4 \pi}+\frac{\mathrm{P}_{1}\left(\tilde{\mathrm{m}}, \mathrm{x}_{\mathrm{O}^{\prime}}, \theta\right)}{4 \pi}\right\} \mathrm{d} \omega=1.0
$$

The particular particle samples were found to fit a general lognormal distribution function:

$$
f(D) d D=\frac{N \delta}{(D-a) \sqrt{\pi}} \exp \left\{-\delta^{2}\left[\ln \left(\frac{D-a}{D_{0}^{-a}}\right)\right]^{2}\right\} d D \frac{\text { particles }}{\mathrm{cm}^{3}-\mathrm{cm}}
$$

with the constraint $a<D<\infty$. The pertinent parameters are the median particle diameter $\dot{\mathrm{D}}_{\mathrm{O}}=0.45$ microns, $\mathrm{a}=0.05$ microns and $\delta=0.896$. 
For our purposes, it is convenient to express the distribution function in terms of mass concentration ( $\mathrm{gms} / \mathrm{cm}^{3}$ ) rather than particle concentration (particles/cm ${ }^{3}$ ). For spherical particles, the particle concentration $\mathrm{N}$ is related to the particle mass concentration $M$ through the equation

$$
N=\frac{6 M}{\pi \rho E(a, \delta)} \text {. }
$$

The parameter $\rho$ is the mass density of the particle material and the function $E(a, \delta)$ is:

$E(a, \delta)=a^{3}+3 a^{2}\left(D_{0}-a\right) \exp \left\{\frac{1}{4 \delta^{2}}\right\}+3 a\left(D_{0}-a\right)^{2} \exp \left\{\frac{1}{\delta^{2}}\right\}+\left(D_{0}-a\right)^{3} \exp \left\{\frac{a}{4 \delta^{2}}\right\}$.

The volume intensity functions for our spherical $\mathrm{S}_{\mathrm{i}_{2}} \mathrm{O}_{2}$ model are then:

$$
\begin{array}{r}
P_{j}\left(\tilde{m}, x_{0}, \theta\right)=\frac{1}{k^{2} \beta_{s}\left(\tilde{m}, x_{o}\right)} \cdot \frac{6 M}{\pi \rho E(a, \delta)} \cdot \frac{\delta}{\sqrt{\pi}} \int_{x_{a}}^{x_{m}} \frac{i_{j}(\tilde{m}, x, \theta)}{\left(x-x_{a}\right)} \exp \left\{-\delta 2\left[\ln \left(\frac{x-x_{a}}{x_{0}-x_{a}}\right)\right]^{2}\right\} d x \\
(j=1,2,3,4)
\end{array}
$$

and the volume scattering coefficient is:

$$
\beta_{s}\left(\tilde{m}, x_{o}\right)=\frac{3}{2} \frac{D_{o}^{2}}{x_{o}^{2}} \cdot \frac{M \delta}{\rho E(a, \delta) \sqrt{\pi}} \int_{x_{a}}^{x_{M}} \frac{x^{2} Q_{s}(\tilde{m}, x)}{\left(x-x_{a}\right)} \exp \left\{-\delta^{2}\left[\ln \left(\frac{x-x_{a}}{x_{o}-x_{a}}\right)\right]^{2}\right\} d x
$$

The mass scattering coefficient is defined as: $k_{S}\left(\tilde{m}, x_{O}\right)=$ $\beta_{S}\left(\tilde{m}, x_{O}\right) / M$ with dimensions of $\mathrm{cm}^{2} / \mathrm{gm}$.

The preceding equations were programmed for the IBM 7094 computer in Fortran IV language. The single particle properties were computed for values of $\mathrm{x}=0(0.2) 120$ for scattering angles $\theta=0^{\circ}(10) 10^{\circ}\left(2^{\circ}\right) 30^{\circ}\left(5^{\circ}\right) 160^{\circ}\left(2^{\circ}\right) 180^{\circ}$. These single particle properties were then integrated over the size distribution function using a step size of $\Delta \mathrm{x}=0.2$. Convergence of the integrals was checked by the program.

\section{III.- SCATTERING MEASUREMENTS}

All of the quantities that are necessary to determine the scattering matrix elements of the particle samples can be measured with a recording Polar Nephelometer. The instrument used in these measurements is a model $\mathrm{PN}-2$ recording Polar Nephelometer manufactured by Spectrametrics Inc. of Burlington, Massachusetts. 
Basically, the instrument consists of a source telescope that is fixed relative to a circular mounting base; a scanning telescope that is mounted on an arm pivotted at the center of the base; and a fixed telescope mounted so that it monitors light scattered at an angle of 11 degrees. The scanning telescope covers scattering angles from 18 degrees to 166 degrees. Light traps are mounted opposite each telescope to provide a uniform black background. Overall diameter of the instrument is 8 feet. The essential features of the instrument are shown in Figs. 1 and 2 .

The detectors used are end-on photomultipliers with $\mathrm{S}-20$ photocathodes. Polarizing filters and spectral filters can be mounted on both of the receivers and on the source. The light source is chopped at $90 \mathrm{cps}$, and synchronous detectors and adjustable time constant integrators are used in both receivers. The optical systems of both the source and the receiver telescopes are identical. The source beam and the field of view of both receivers are rectangular, roughly 2 inches high and one-half inch wide. The intersection of the source beam and the receiver field of view defines the common volume. As the scanning arm moves, the volume changes size and a correction for this effect is made during data reduction. The time required to scan through the complete range of scattering angles can be varied from 1 to over 20 minutes.

\section{Calibration of the Polar Nephelometer system}

The system was calibrated in absolute quantities by comparing the intensity of the light scattered from the particles in the common volume with the intensity reflected or transmitted from a calibration standard. The method is identical to that used by Pritchard and Elliot (ref. 2). Since a complete discussion and proof of the method are given in this reference, only a qualitative description is given here. Briefly, a calibration standard made of thin plastic sheet of measured reflectance and transmittance is placed in the common volume perpendicular to the scattering plane. The calibration screen is moved slowly in a direction perpendicular to the screen surface so that each pair of rays common to the source and receiver beams is intercepted during the screen's travel. The intensity of the light reflected by the screen is recorded as it moves, and the integral of the signal over the distance travelled is recorded. This integral is compared to the intensity of light scattered by the particle samples. The reflection and transmission matrices of the calibration screen are measured and compared to an absolute standard; a glass plate covered with magnesium oxide smoke particles. The reflection matrix of the MgO standard has been measured by Pritchard and Elliot (ref. 2). 


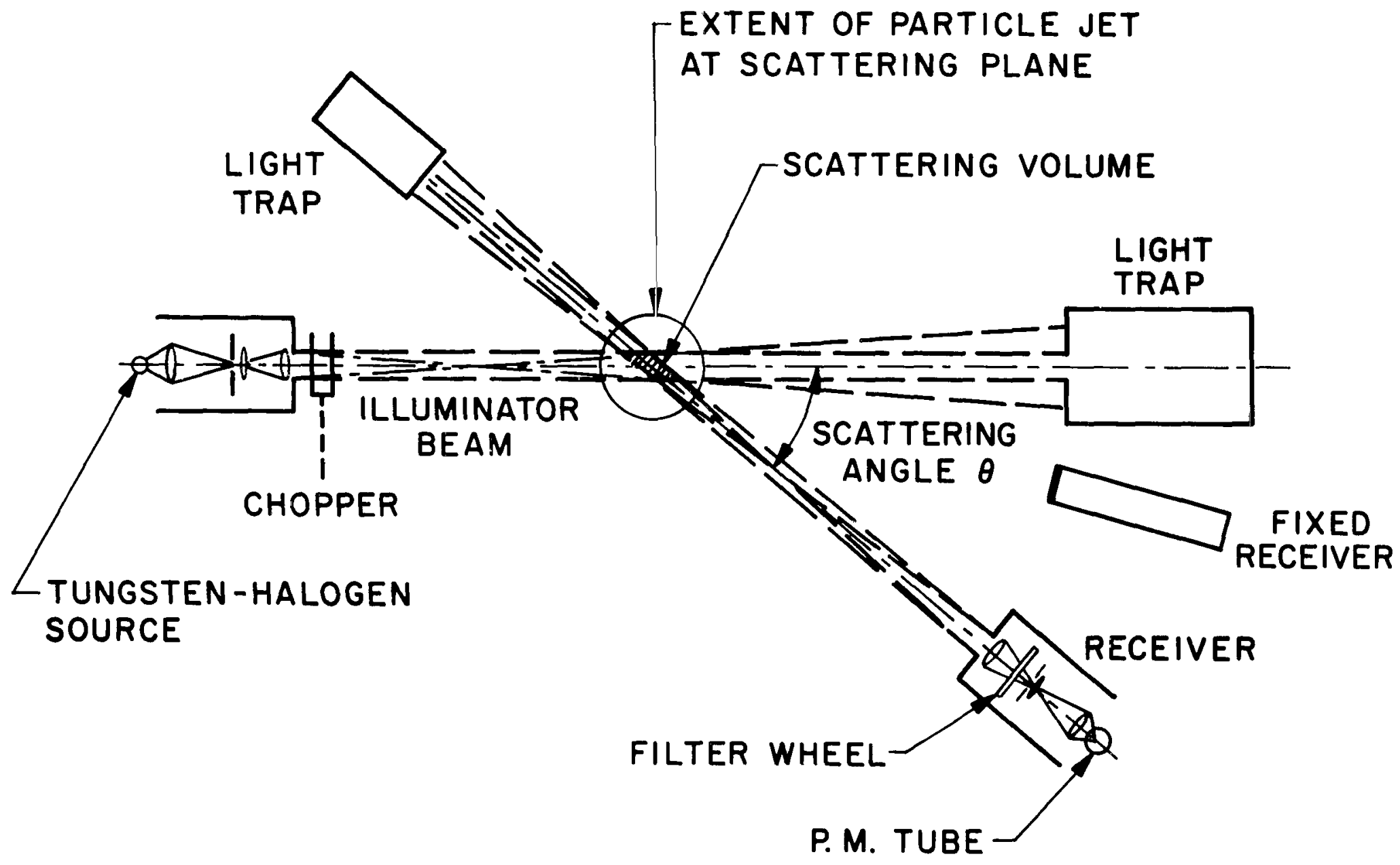




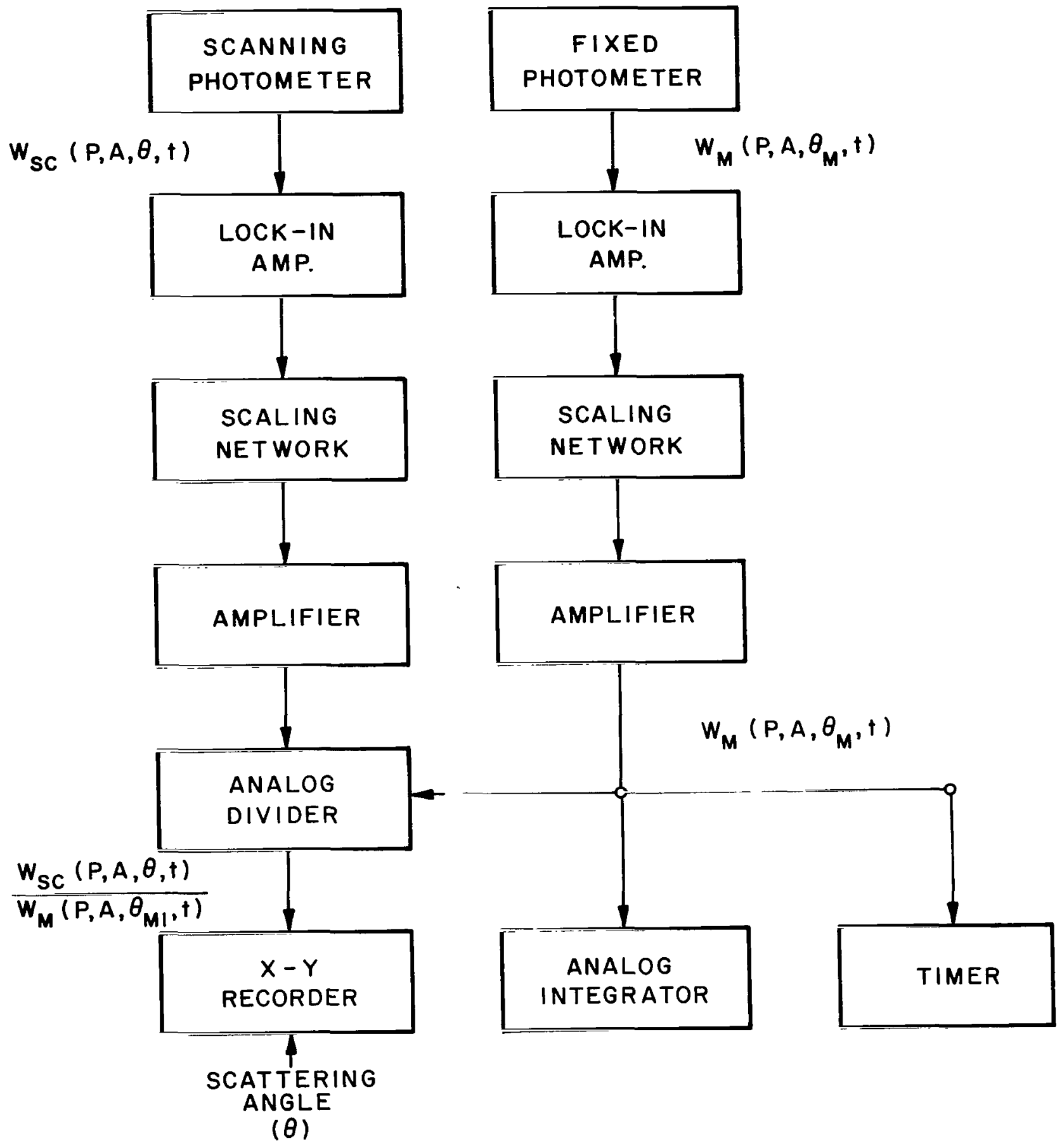

Figure 2.- Block diagram of signal processing system 
The calibration was carried out for every combination of scattering angle, polarizing and analyzing filters, and color filters used in the measurements. The procedure yields a calibration constant $K(P, A, \theta ; \lambda)$ such that the volume scattering coefficient for the sample volume is:

$$
\beta_{S}(P, A, \theta ; \lambda)=K(P, A, \theta ; \lambda) \times W_{S C}(P, A, \theta ; \lambda)
$$

where $W_{S C}(P, A, \theta ; \lambda)$ is the output signal from the scanning photometer channel for source polarizer, P; receiver analyzer A at scattering angle $\theta$; and effective wavelength $\lambda$. The effective wavelength is the center wavelength of the combination of source spectrum, filter transmittance and photocathode response.

\section{Particle Dispersion System}

The particles are carried through the common volume by a jet of dry nitrogen gas. The jet axis coincides with the axis of rotation of the scanning arm. An exhaust system scavenges the spent particles through the hollow hub of the scanning arm. The particles, because of their small sizes, take up the motion of the gas stream and the jet can be described as a two-phase gas jet. The mass fraction of particles in the stream is small enough so that their presence does not effect the flow properties materially.

The particle density in the common volume was determined by surveying the velocity distribution in the jet at a sufficient number of points along the jet axis and radially from the axis. The velocity measurements were made both with and without particles in the stream. No essential velocity differences were noted. Then, associating the particle mass concentration in the jet with ordinary gas mixing rates and employing subsonic, two-phase, flow theory (ref. 10), the particle density distribution throughout the jet was calculated. A second method was used to substantiate the first calibration. Photographs of the jet illuminated by parallel light were taken and processed with a Joyce-Liebl microdensitometer. The density distribution in the jet was then determined using a standard Abel inversion (ref. 11). The relative density distributions obtained were identical to those given by the velocity measurements. With the jet calibration completed, the mean particle mass concentration in the common volume can be expressed in terms of the axial velocity of the jet at the center of the common volume and mass flow rate in the jet. Since the size of the common volume changes with scanning angle, the mean density of the particles in the volume changes and a correction must be applied during the data reduction. 


\section{Particle Characteristics}

For these experiments we used the Pennsylvania Glass Sand Company's "5 micron minusil" particles. According to the manufacturer's specifications, the material is a high-purity crystalline silica (99.9\% $\left.\mathrm{SiO}_{2}\right)$ with a specific gravity of 2.65 and a refractive index of 1.55 for visible wavelengths. The particles have a flat plate-like shape with irregular edges. some typical particles are shown in Fig. 3 .

The particle size distribution was determined by sampling the air-particle jet at the common volume. Electron micrographs were prepared from the samples and the size distribution was measured with a Zeiss Particle Size Analyzer, which permits measurement of particle size frequency and cumulative size distribution. Both functions were measured for our samples using well over 6,000 particles for each function. The data were found to fit a general lognormal function. The particle frequency function and cumulative distribution function are shown in Figs. 4 and 5 .

\section{IV.- SIGNAL PROCESSING AND DATA REDUCTION}

The jet containing the particles is turbulent, and fluctuations in the instantaneous particle concentration result in fluctuations in the recorded signal. To eliminate these fluctuations, the fixed photometer was used to normalize the scan photometer signal. A block diagram of the signal processing system is shown in Fig. 2. The signal from the scanning photometer passes directly into a synchronous detecter or lock-in amplifier set at the chopping frequency. The signal is then amplified and scaled. A similar system processes the signal from the monitor photometer. Both signals are then fed into an analog divider whose output is a scaled quotient:

$$
\text { const } x W_{S C}(P, A, \theta) / W_{M}\left(P, A, \theta_{M}\right)
$$

The output signal from the divider is displayed on one axis of an $\mathrm{x}-\mathrm{y}$ recorder against scan angle. The scan angle signal is provided by a ten-turn, high-precision potentiometer driven by the scan arm. In addition, the monitor signal is integrated. over the running time of the jet, and the resulting integral and jet running time are recorded.

Since the monitor is fixed at a scattering angle of 11 degrees, fluctuations in its signal are only due to jet density fluctuations (assuming the character of the particles in the jet remain constant). Integrating the monitor signal over the total running time $T$ of the jet we have: 


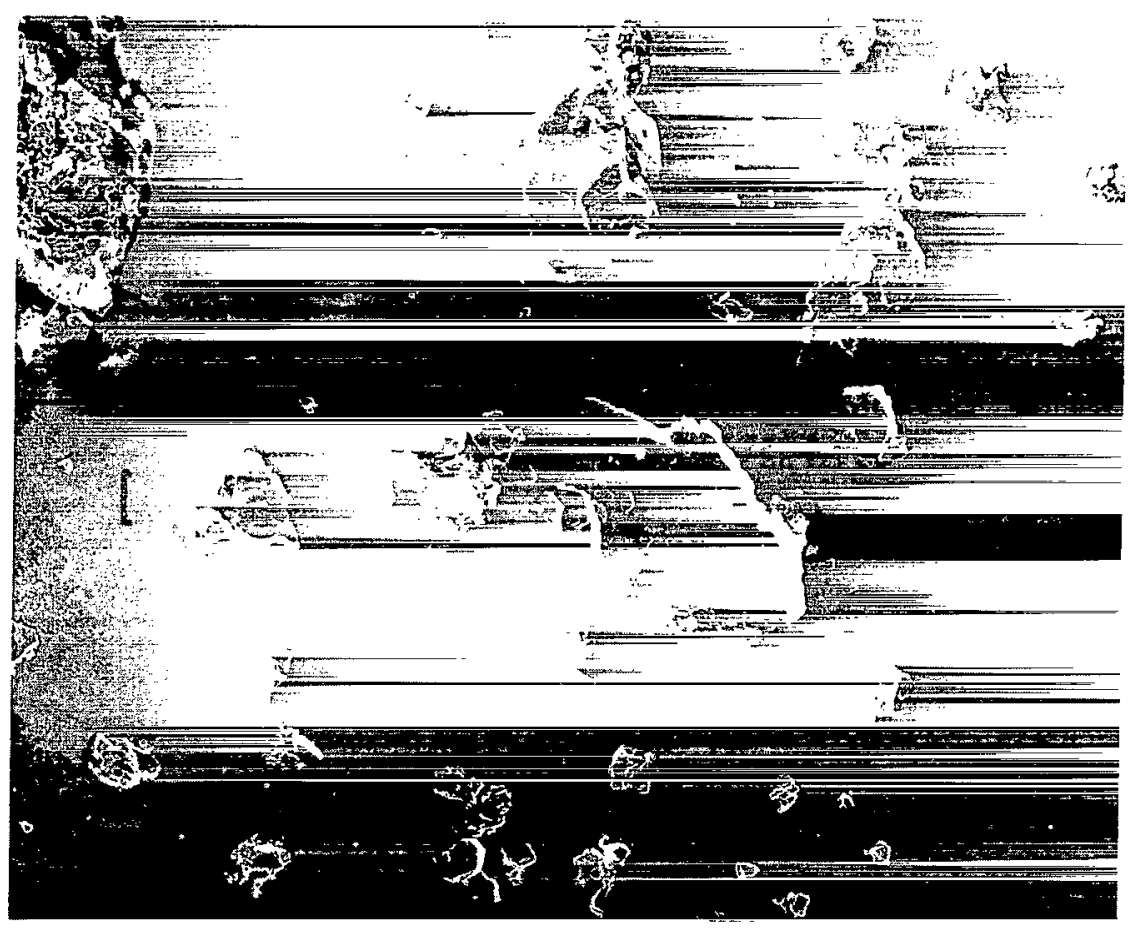

Figure 3.- Typical "5-micron minusil" particles

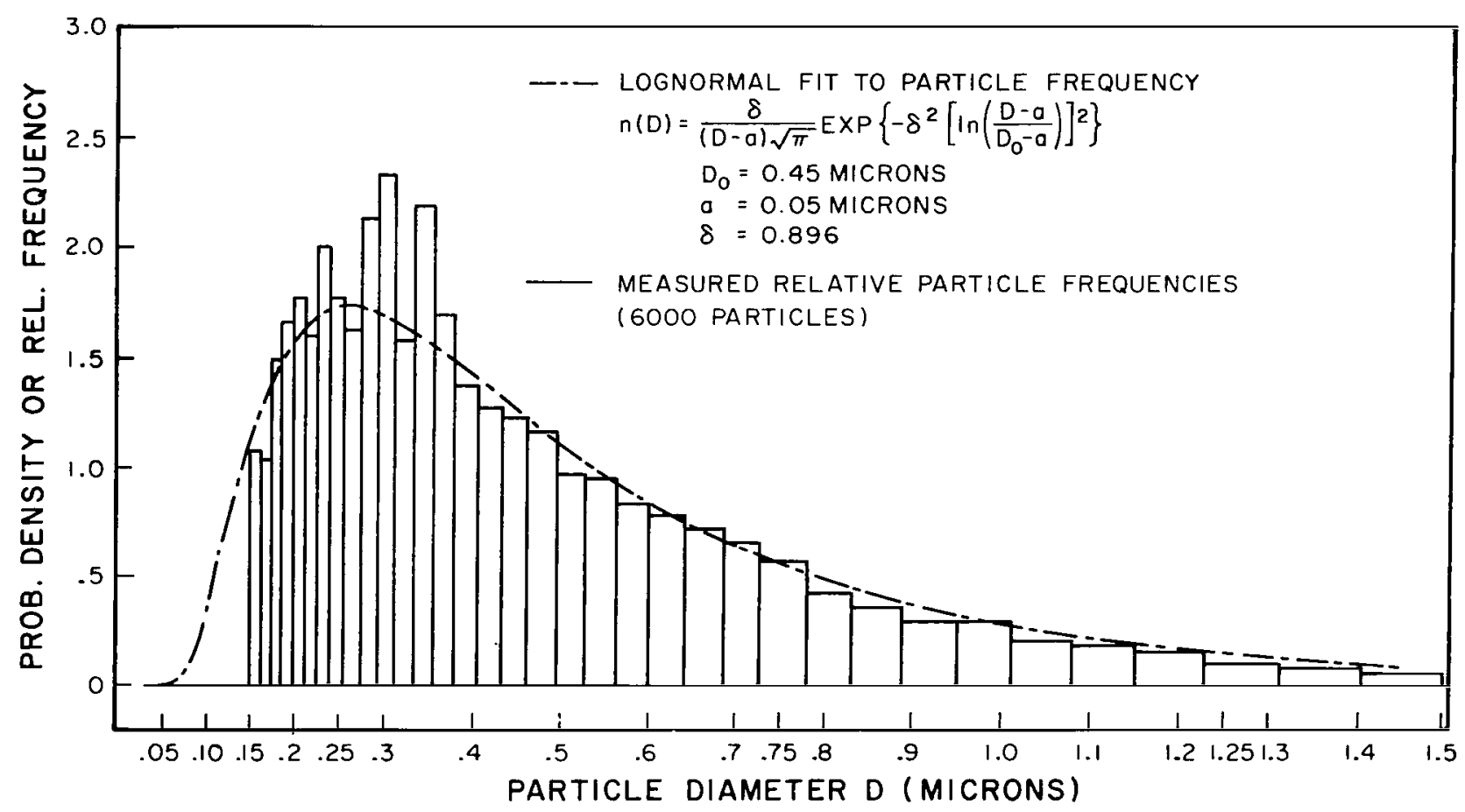

Figure 4.- Minusil particle density function 


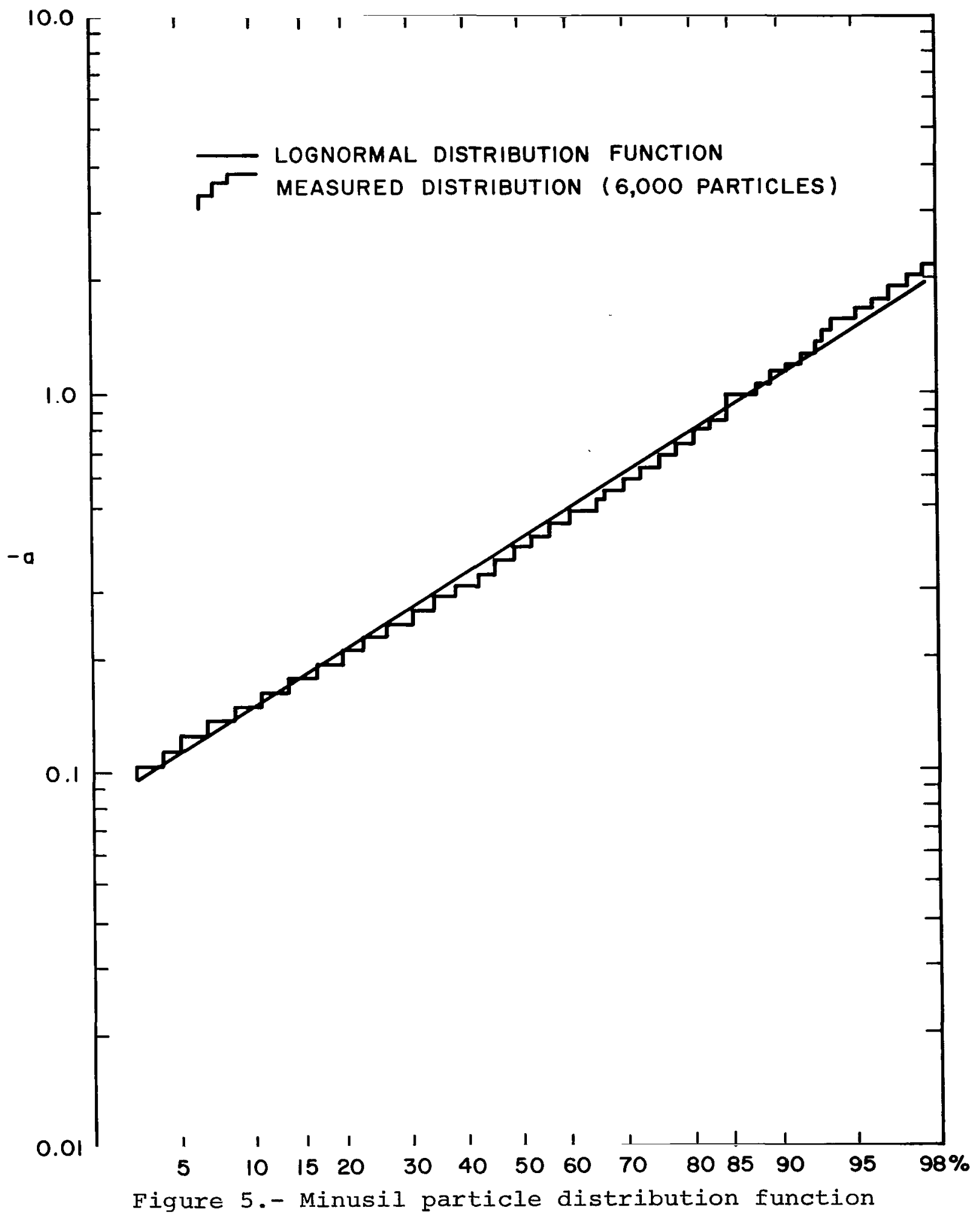




$$
V \equiv \int_{0}^{t} W_{M}\left(P, A, \theta_{M}, t\right) d t
$$

The mass scattering coefficient for the sample is:

$k_{S}\left(P, A, \theta_{M}\right) \equiv \beta_{S}\left(P, A, \theta_{M}\right) / \tilde{\rho}\left(\theta_{M}, t\right)=W_{M}\left(P, A, \theta_{M}, t\right) K\left(P, A, \theta_{M}\right) / \tilde{\rho}\left(\theta_{M}, t\right)$

where $\tilde{\rho}\left(\theta_{M}\right)$ denotes the mean mass density of particles in the common volume at scattering angle $\theta$ and $K\left(P, A, \theta_{M}\right)$ is the system calibration constant.

Then

$$
V=\frac{k_{S}\left(P, A, \theta_{M}\right)}{K\left(P, A, \theta_{M}\right)} \int_{0}^{t} \tilde{\rho}\left(\theta_{M}, t\right) d t .
$$

The jet calibration procedure yields a relation between the particle mass density in the common volume and the overall mass flow rate $\dot{M}(t)$ in the jet:

$$
\tilde{\rho}(\theta, t)=C(\theta) \dot{M}(t) \text {. }
$$

Then

$$
\begin{aligned}
v & =\frac{k_{S}\left(P, A, \theta_{M}\right)}{K\left(P, A, \theta_{M}\right)} \times C\left(\theta_{M}\right) \int_{O}^{T} \dot{M}(t) \\
& =\frac{k_{S}\left(P, A, \theta_{M}\right)}{K\left(P, A, \theta_{M}\right)} \times C\left(\theta_{M}\right) M
\end{aligned}
$$

where $M$ is the total mass of particles expended during the run. Then the mass scattering coefficient for the monitor is:

$$
k_{S}\left(P, A, \theta_{M}\right)=v \times k\left(P, A, \theta_{M}\right) / M x C\left(\theta_{M}\right), \frac{\mathrm{cm}^{2}}{g m-s t e r} .
$$

Once the monitor scattering coefficient is known the divided signal can be reduced since:

$$
\frac{W_{S C}(P, A, \theta, t)}{W_{M}\left(P, A, \theta_{M}, t\right)} \propto \frac{k_{S}(P, A, \theta)}{k_{S}\left(P, A, \theta_{M}\right)} \times \frac{K\left(P, A, \theta_{M}\right)}{K(P, A, \theta)} \times \frac{\tilde{\rho}(\theta, t)}{\tilde{\rho}\left(\theta_{M}, t\right)}
$$

or 


$$
\bar{k}_{k_{s}} \frac{(P, A, \theta)}{\left(P, \bar{A},{ }_{M}\right)}=\text { const } \times \frac{W_{S C}(P, A, \theta, t)}{W_{M}\left(P, \bar{A}, \theta_{M}, t\right)} \times \frac{K(P, A, \theta)}{K\left(P, \bar{A}, \theta_{M}\right)} \times \frac{C\left(\theta_{M}\right)}{C(\theta)} .
$$

These equations are the basis for the data reduction. All of the constants needed are derived from calibration of the jet and the nephelometer system.

The preceding analysis assumes that only single scattering occurs in the common volume during the measurements. Single scattering was proved experimentally by taking data at different mass flow rates and confirming a linear relation between signals observed and mass flow. Additionally, calculations based on the measured mass scattering coefficients and particle densities confirmed that the optical thickness through the jet was always small enough so that only single scattering was possible $(\tau<0.15)$.

\section{Derivation of Matrix Elements}

The stokes vector of the light scattered from the sample volume at any angle $\theta$ and accepted by the analyzer in the photometer can be written in matrix form as:

$$
\left[\begin{array}{l}
I_{s} \\
Q_{s} \\
U_{s} \\
V_{s}
\end{array}\right]=\operatorname{const}\left[\begin{array}{l}
A_{i j} \\
s_{i j}
\end{array}\right]\left[\begin{array}{l}
P_{i j} \\
\\
0 \\
0
\end{array}\right]
$$

The symbols $\left[\mathrm{A}_{i j}\right],\left[\mathrm{P}_{i j}\right]$, and $\left[\mathrm{S}_{i j}\right]$ denote the $4 \times 4$ Mueller matrices describing the photometer analyzer, light source polarizer and the scattering medium. The elements of the scattering matrix are functions of the particle size, shape, and material, as well as the angle between the incident and scattered beams. Only the first stokes parameter of the scattered light can be measured by our photometer. However, by making measurements with suitable combinations of polarizers and analyzers, each of the sixteen possible matrix elements can be obtained. In these measurements, only linear and right circular polarizers are used. The polarizers were $H, V, D, d$, and $R$. The usual sign convention is used here. If one looks along the light beam in the direction of propagation, the symbol $\mathrm{H}$ denotes polarization parallel to the scattering plane of the instrument and $V$ denotes perpendicular polarization. The symbol $D$ denotes polarization at 45 degrees to the horizontal with the electric vector pointing upward and to the right; $d$ is orthogonal to $D$, and $R$ denotes clockwise rotation about the direction of propa- 
gation when looking in the direction of propagation. For the polarizer, the symbols indicate the polarization form transmitted, while for the analyzer, they denote the polarization form accepted. For these measurements, the following combinations were used:

$$
\begin{aligned}
& \mathrm{UU}, \mathrm{HH}, \mathrm{VV}, \mathrm{VH}, \mathrm{HV}, \mathrm{DD}, \mathrm{dd}, \mathrm{Dd}, \mathrm{dD}, \mathrm{RR}, \\
& \mathrm{Rd}, \mathrm{dR}, \mathrm{DR}, \mathrm{RD}, \mathrm{HD}, \mathrm{DH}, \mathrm{HR} \& \mathrm{RH} .
\end{aligned}
$$

In addition, consistency checks were made between various measured and computed values. For example, for an unpolarized source and receiver:

$$
\mathrm{k}_{\mathrm{S}}(\mathrm{U}, \mathrm{U}, \theta)=\frac{\mathrm{I}_{\mathrm{s}}}{\mathrm{I}_{\mathrm{O}}}(\mathrm{U}, \mathrm{U}, \theta)=\mathrm{S}_{11}\left(\tilde{\mathrm{m}}, \mathrm{x}_{\mathrm{O}}, \theta\right)
$$

while some matrix manipulations give

$$
\mathrm{S}_{11}\left(\tilde{\mathrm{m}}, \mathrm{x}_{\mathrm{O}}, \theta\right)=\frac{1}{4}\left[\mathrm{k}_{\mathrm{S}}(\mathrm{H}, \mathrm{H}, \theta)+\mathrm{k}_{\mathrm{S}}(\mathrm{V}, \mathrm{V}, \theta)+\mathrm{k}_{\mathrm{S}}(H, \mathrm{~V}, \theta)+\mathrm{k}_{\mathrm{s}}(\mathrm{V}, \mathrm{H}, \theta)\right]
$$

Cross checks of this type were made whenever possible and were found to be satisfied within our experimental error.

The measurements confirmed that the following relations held between the off-diagonal matrix elements for all scattering angles: $\mathrm{S}_{12}=\mathrm{S}_{21}, \mathrm{~S}_{34}=-\mathrm{S}_{43}$, and $\mathrm{S}_{13}=-\mathrm{S}_{31}=\mathrm{S}_{14}=\mathrm{S}_{41}=$ $\mathrm{S}_{23}=-\mathrm{S}_{32}=\mathrm{S}_{24}=\mathrm{S}_{42}=0$. The scattering matrix then has six independent non-zero elements: the condition for a symmetric, isotropic scattering medium.

\section{V.- RESULTS AND DISCUSSION}

Our results are summarized in Figs. 6 through 9. The matrix elements $S_{11}\left(\tilde{m}, x_{0}, \theta\right)$ and $S_{22}\left(\tilde{m}, x_{0}, \theta\right)$ are shown in $F i g .6$ as functions of scattering angle. For comparison, the corresponding matrix elements for spherical particles with the same size distribution are also shown. For spherical particles the two elements $S_{11}$ and $S_{22}$ are equal. The experimental points shown represent averages of at least six distinct measurements. The flags represent one standard deviation of the data about the mean values. The mass scattering coefficient for unpolarized light (matrix element $s_{11}\left(\tilde{m}, x_{0}, \theta\right)$ shows excellent agreement with the theoretical predictions for scattering angles of less than 80 degrees. For larger scattering angles, the theory disagrees with the measurements. At backscattering angles, there is a full order of magnitude difference. These results confirm our earlier measurements for unpolarized light (ref. 4). The better agreement obtained here for scattering angles less than 80 degrees must be in part due to our improved knowledge of the particle 


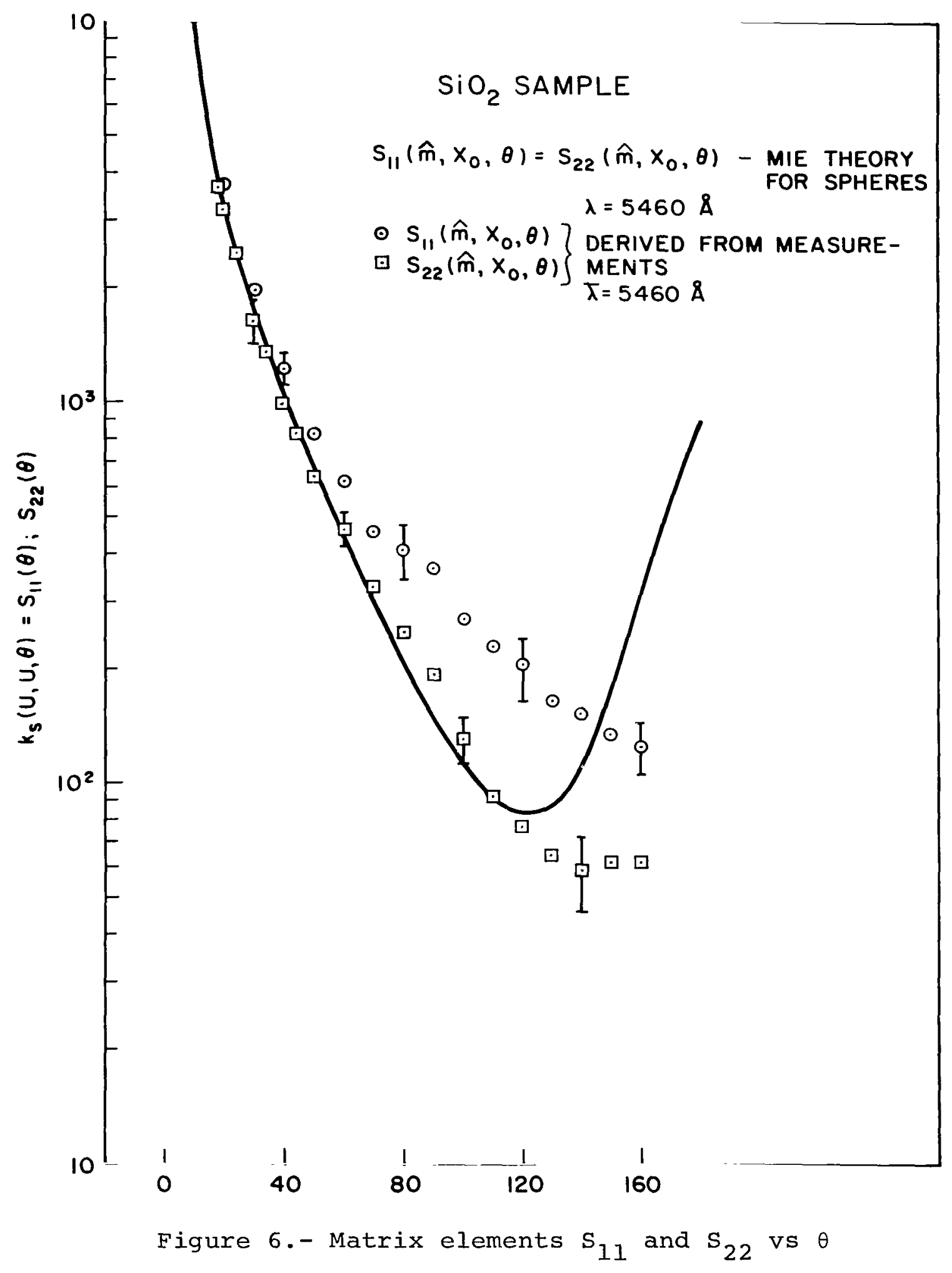


sizes used in the experiment and our calibration procedure.

The fact that the measured matrix elements $S_{11}\left(\tilde{m}, x_{0}, \theta\right)$ do not equal the element $\mathrm{S}_{22}\left(\tilde{\mathrm{m}}, \mathrm{x}_{\mathrm{O}}, \theta\right)$ is not surprising. That particular relation holds only for spherical particles, and the experimental data merely reflect the fact that randomly oriented irregular discs do not resemble spheres in every respect. In fact, recalling the more fundamental amplitude scattering matrix from which the intensity scattering matrix is derived (ref. 6), we have:

$$
\left[\begin{array}{l}
E_{s} l \\
E_{s} r
\end{array}\right]=\text { const } \times\left[\begin{array}{ll}
A_{2} & A_{3} \\
A_{4} & A_{1}
\end{array}\right]\left[\begin{array}{l}
E_{0} l \\
E_{0} r
\end{array}\right]
$$

when the reference plane and subscripts are as described earlier. For spheres, the amplitude scattering functions $A_{3}$ and $A_{4}$ are equal to zero. Only then do the scattering matrix elements, $\mathrm{S}_{11}=\mathrm{S}_{22}$ and $\mathrm{S}_{33}=\mathrm{S}_{44}$. Our results merely confirm that the amplitude matrix elements $A_{3}$ and $A_{4}$ are not zero in this case. The better agreement with the theoretical curve exhibited by $S_{22}$ is puzzling. We have no explanation for this fact at this time.

The measured matrix elements $\mathrm{s}_{12}\left(\tilde{\mathrm{m}}, \mathrm{x}_{\mathrm{O}}, \theta\right)$ and $\mathrm{s}_{21}\left(\tilde{\mathrm{m}}, \mathrm{x}_{\mathrm{O}}, \theta\right)$ are shown in Fig. 7 as functions of scattering angle $\theta$. These elements were found to be equal within experimental accuracy. The corresponding matrix element for the spherical particle model is shown for comparison. The measured results show a preponderance of vertical scattering (negative values for matrix element S12), while the spherical model predicts a preponderance of horizontal scattering. These measurements show the same general behavior as the measurements on atmospheric samples reported by Rozenberg and Gorchakov (ref. 12).

Fig. 8 shows the measured scattering matrix elements $\mathrm{S}_{33}\left(\tilde{\mathrm{m}}, \mathrm{x}_{0}, \theta\right)$ and $\mathrm{S}_{44}\left(\tilde{\mathrm{m}}, \mathrm{x}_{0}, \theta\right)$ as functions of scattering angle $\theta$. Again, the fact that these elements are not equal implies, as in the case of the first two diagonal elements, that the amplitude functions $\mathrm{A}_{3}, \mathrm{~A}_{4}$ are non-zero. The close agreement of the measured elements to the theoretical prediction is encouraging, since the element $s_{33}\left(\tilde{\mathrm{m}}, \mathrm{x}_{0}, \theta\right)$ shows, at least in the Mie theory, much more sensitivity to index of refraction than does $s_{11}$ or $S_{22}$.

Finally, the off-diagonal matrix elements $\mathrm{S}_{34}\left(\tilde{\mathrm{m}}, \mathrm{x}_{\mathrm{O}}, \theta\right)=$ $-\mathrm{S}_{43}\left(\tilde{\mathrm{m}}, \mathrm{x}_{\mathrm{O}}, \theta\right)$ is shown in Fig. 9. The equality of these two matrix elements was confirmed within our experimental accuracy. Again, the theoretical values for spherical particles are shown for comparison. The agreement in this case is somewhat poorer than for the diagonal elements. 


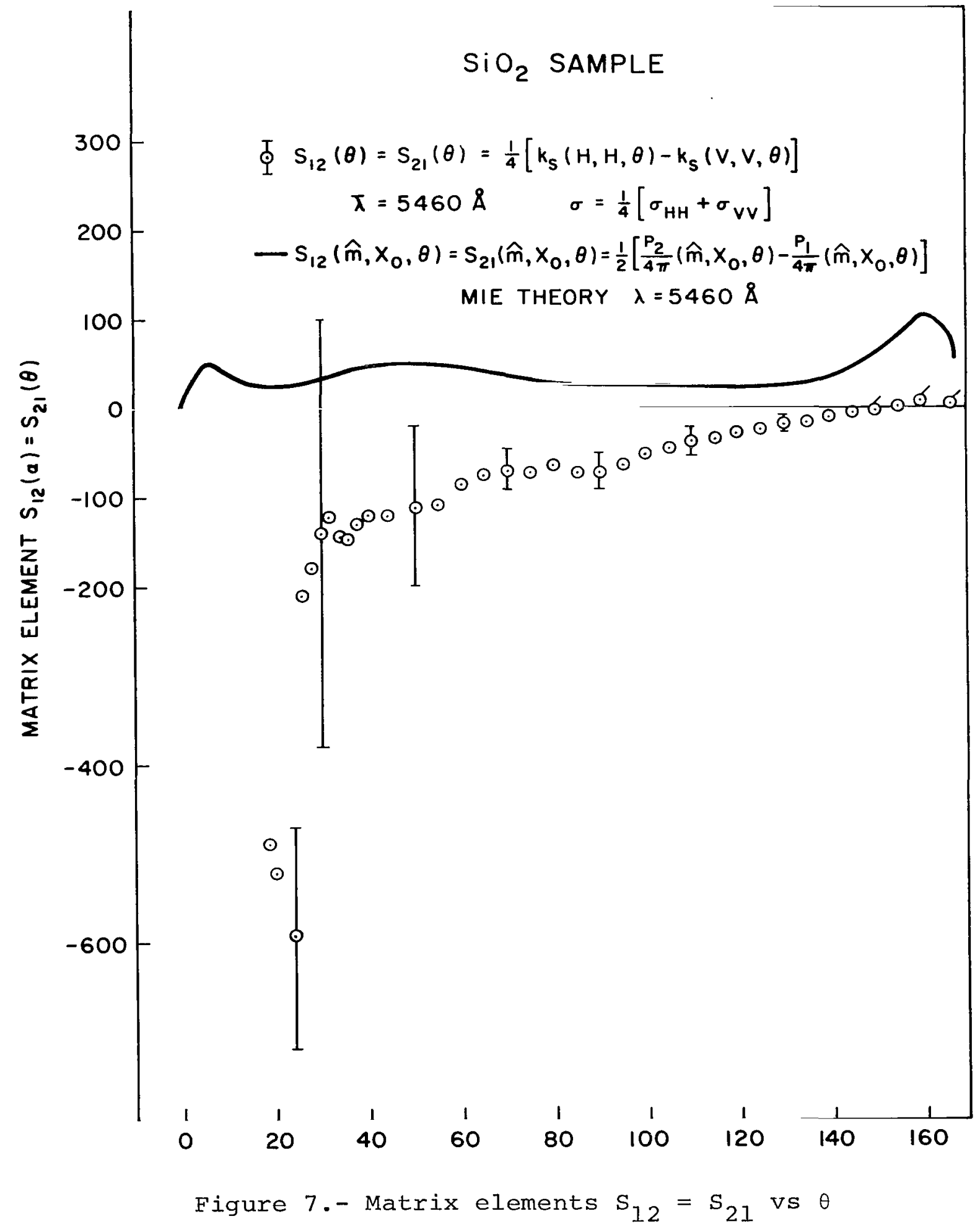




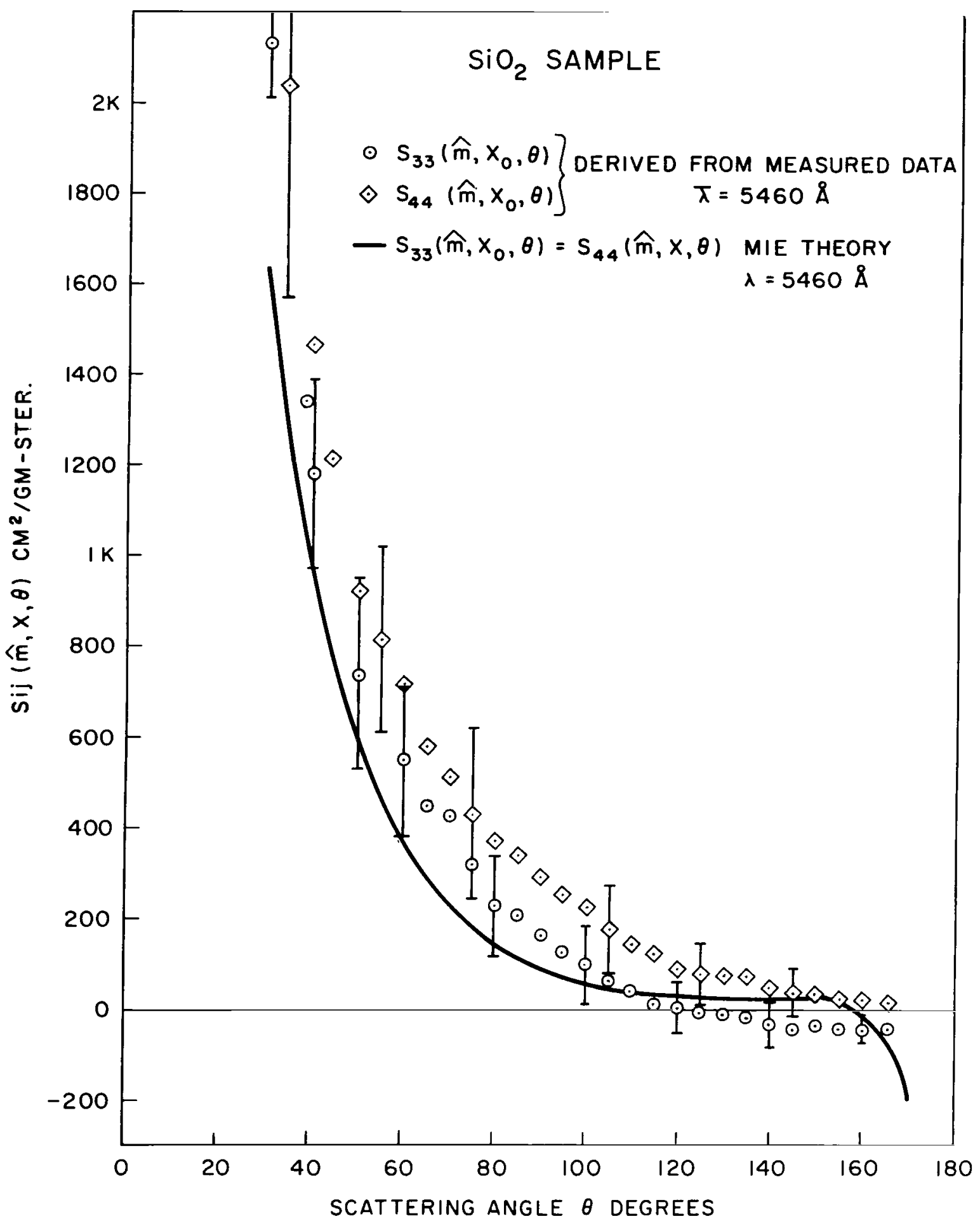

Figure 8.- Matrix elements $S_{33}$ and $s_{44}$ vs $\theta$ 


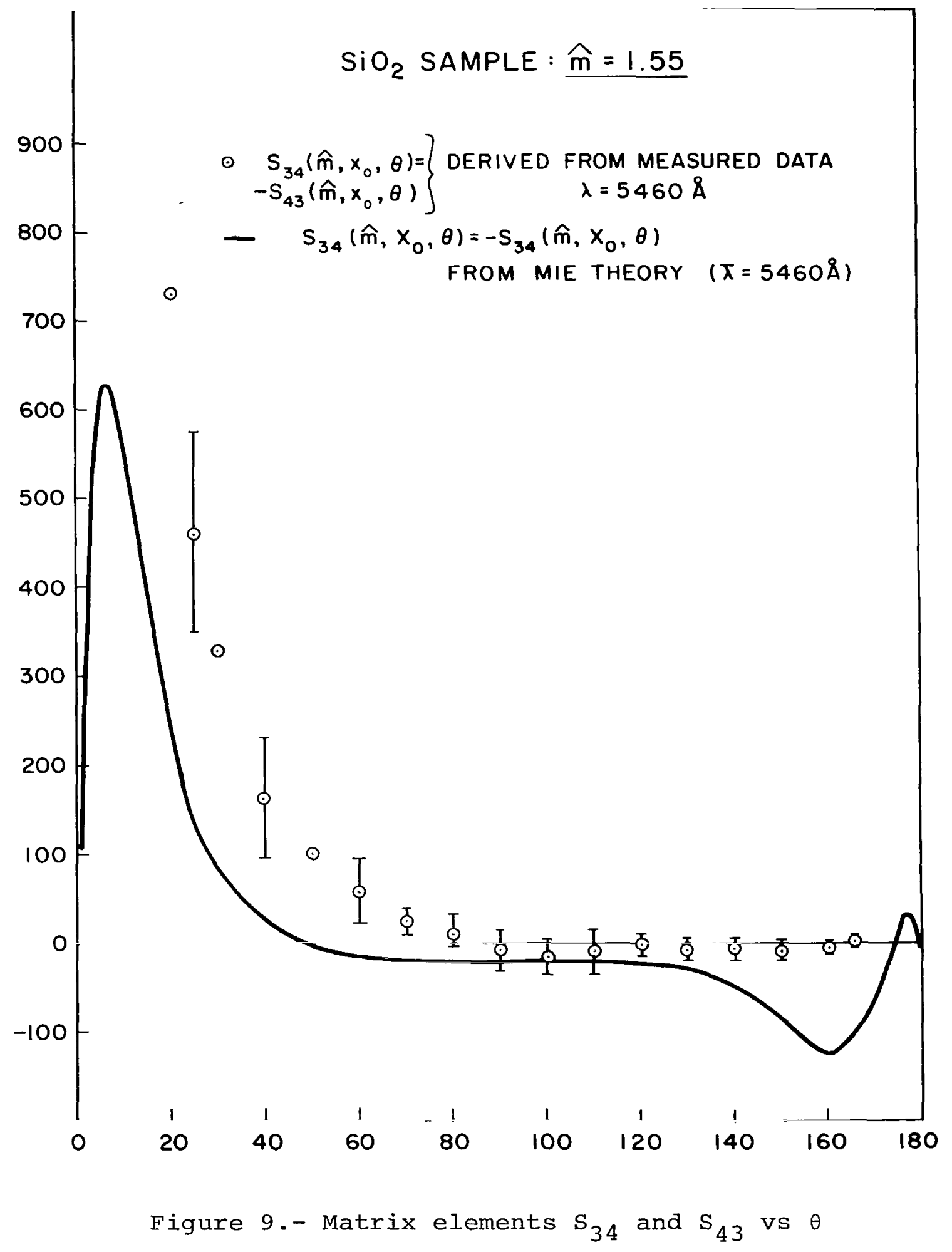


It is tempting to try to generalize from these limited results. However, the data presented here represent measurements on one particle material and shape, fitting one size distribution and at one effective wavelength. Generalizations will require much more information. At present, measurements are being made at other wavelengths and the entire set of measurements will be repeated for the same particle material and shape but with larger sizes. Samples of the material are available with three larger size ranges. Measurements of the scattering matrix elements for other particle materials and shapes are also planned.

There are several conclusions to be drawn from these results, however. First, that the unpolarized mass scattering coefficient for a polydisperse system of irregular but randomly oriented particles shows a remarkable similarity to the corresponding coefficient for spherical particles, but that predicting backscattering for such particles on the basis of the spherical model can lead to serious errors. Second, that the particle dispersion system used in these experiments generates a scattering medium whose matrix exhibits the properties of an isotropic symmetric medium. 
REFERENCES

1. Deirmendjian, D.: Scattering and Polarization Properties of Polydispersed Suspensions with Partial Absorption. Proc. of the Interdisciplinary Conference on Electromagnetic Scattering, Potsdam, New York, 1962 (Pergamon Press Ltd., London 1963).

2. Pritchard, B.S.; and Elliott, W.G.: Two Instruments for Atmospheric Optics Measurements. J. Opt. Soc. Am., vol. 50, no. 3, 1960, p. 191 .

3. Hodkinson, J.: Light Scattering and Extinction by Irregular Particles Larger than the Wavelength. Proc. of the Interdisciplinary Conference on Electromagnetic Scattering. Potsdam, New York, 1962 (Pergamon Press, Ltd., London, 1963).

4. Holland, A.C.; and Draper, J.S.: Analytical and Experimental Investigation of Light Scattering from Polydispersions of Mie Particles. Applied Optics, vol. 6, 1967, p. 511.

5. Perrin, F.: Polarization of light scattered by Isotropic Opalescent Media. Journal of Chemical Physics, vol. 10, 1942, pp. 414-427.

6. Van De Hulst, H.C.: Light Scattering by Small Particles. John Wiley and Sons, New York, 1957.

7. Rozenberg, G.V.: Light Scattering in the Earth's Atmosphere. Soviet Physics Uspekhi, vol. 3, no. 3, Nov.-Dec., 1960.

8. Parke, N.G.: Statistical Optics: Mueller Phenomenological Algebra. M.I.T. Research Lab. of Electronics, Rept. 119, June, 1949.

9. Chandrasekhar, S.: Radiative Transfer. Dover Publications, New York, 1960.

10. Abramovich, G.N.: The Theory of Turbulent Jets. M.I.T. Press, Cambridge, Mass. 1963.

11. Cremers, C.J. and Birkebak, R.C.: Application of the Abel Integral Equation to Spectrographic Data. Applied Optics, vol. 5, no. 6, June 1966 , p. 1057 .

12. Rozenberg, G.V., and Gorchakov, G.: Measurements of the light Scattering Matrix in the Atmospheric Surface Layer. Izvestiya, Atm. and Oceanic Phys., vol. 1, no. W12, 1965, pp. $1279-88$. 


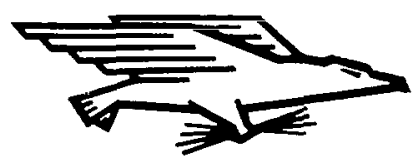

POSTAGE AND FEES PAID NATIONAL AERONAUTICS AND SPACE ADMINISTRATION

\author{
$04 \mathrm{~L} 0014951305 \quad 0932100903$ \\ AIR FURCE WERPCWS LABCRATURYTMLIL/ \\ KIRTIANO AIR FORCE BASE, IEA MEXICU $\$ 7111$

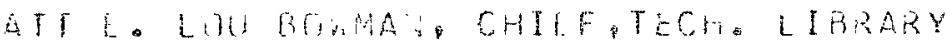

POSTMASTER: If Undeliverable ( Section 158 Postal Manual) Do Nor Return

"The acronatitical and space actitities of the United States shall be conducted so as to contribute. . . to the expansion of buman knowledge of phenomena in the atmospbere and space. The Administration shall protide for the uidest practicable and appropriate dissemination of information concerning its actinities and the results thereof."

- National Aeronautics and Space Act of 1958

\title{
NASA SCIENTIFIC AND TECHNICAL PUBLICATIONS
}

TECHNICAL REPORTS: Scientific and technical information considered important, complete, and a lasting contribution to existing knowledge.

TECHNICAL NOTES: Information less broad in scope but nevertheless of importance as a contribution to existing knowledge.

TECHNICAL MEMORANDUMS:

Information receiving limited distribution because of preliminary data, security classification, or other reasons.

CONTRACTOR REPORTS: Scientific and technical information generated under a NASA contract or grant and considered an important contribution to existing knowledge.
TECHNICAL TRANSLATIONS: Information published in a foreign language considered to merit NASA distribution in English.

SPECIAL PUBLICATIONS: Information derived from or of value to NASA activities. Publications include conference proceedings, monographs, data compilations, handbooks, sourcebooks, and special bibliographies.

\section{TECHNOLOGY UTILIZATION}

PUBLICATIONS: Information on technology used by NASA that may be of particular interest in commercial and other non-aerospace applications. Publications include Tech Briefs, Technology Utilization Reports and Notes, and Technology Surveys.

Details on the availability of these publications may be obtained from:

\author{
SCIENTIFIC AND TECHNICAL INFORMATION DIVISION \\ NATIONAL AERONAUTICS AND SPACE ADMINISTRATION \\ Washington, D.C. 20546
}

